\title{
Homotopy operators for the variational bicomplex, representations of the Euler-Lagrange complex, and the Helmholtz-Sonin conditions
}

\author{
M. Crampin \\ Department of Mathematical Physics and Astronomy, Ghent University \\ Krijgslaan 281, B-9000 Gent, Belgium \\ and D. J. Saunders \\ Department of Algebra and Geometry, Palacky University, Olomouc \\ 77900 Olomouc, Czech Republic
}

July 28,2008

\begin{abstract}
We give formulæ for two distinct local homotopy operators for the horizontal differential in the variational bicomplex. We deduce two different representations of the classes of forms in the Euler-Lagrange complex, and hence two different versions of the Helmholtz-Sonin equations for the local variationality of a source form. We give explicit relationships between these two versions of the equations.
\end{abstract}

\section{Introduction}

A significant step in the development of the differential-geometric theory of the calculus of variations was taken when it was realised that the operation of deriving the EulerLagrange equations from a Lagrangian form could be embedded in a complex (in the sense of homological algebra), the so-called Euler-Lagrange complex or variational sequence. Such problems as determining necessary and sufficient conditions for a system of differential equations to be variational (that is, to be the Euler-Lagrange equations of some Lagrangian) and describing variationally trivial or null Lagragians (those whose Euler-Lagrange equations vanish identically) have a natural place in the context of the Euler-Lagrange complex. A further significant step was taken when it was realised that the Euler-Lagrange complex can in turn be embedded in a bicomplex, the so-called variational bicomplex. The properties of the Euler-Lagrange complex are thereby seen to derive ultimately from those of the bicomplex and its maps, the horizontal and vertical differentials. A recent comprehensive discussion of the bicomplex, together with an 
account of the history of the subject and an extensive list of references, may be found in Vitolo's survey article in the Handbook of Global Ananlysis [18]. An older, but very useful and sometimes more detailed, standard reference is Anderson's still unpublished book, which is readily available from his website [1].

One early difficulty in setting up the variational bicomplex lay in finding a satisfactory proof of the local exactness of the horizontal differential. Various authors have by now given proofs [15, 16, 17], and in [1] Anderson gives an extensive description of the theory using the technique of Euler operators. We use instead the Frölicher-Nijenhuis theory of derivations [4] to give explicit formulæ for two new homotopy operators for the horizontal differential and to prove the homotopy property. We believe that by comparison with other efforts our formulæ are both approachable and elegant; however, the main interest of our paper derives from the duplication.

A local homotopy operator for the variational bicomplex can be used to construct a local representation of the Euler-Lagrange complex, and so corresponding to our two homotopy operators we have two representations of this complex. In particular, the space of representative $(m+2)$-forms (where $m$ is the number of independent variables) differs in the two cases, and so the condition for an $(m+1)$-form to be variational takes different forms. That is to say, the two representations produce different versions of the Helmholtz-Sonin conditions - versions which turn out to be markedly different in appearance and properties. We shall give explicit formulæ for the two versions and the relation between them.

(As an aside, we should point out that representations of the Euler-Lagrange complex can also be obtained from the finite-order variational sequence instead of from the variational bicomplex, which is defined on a bundle of infinite-order jets. The finite-order sequence was introduced in [8], and a description of a representation in this context is given in [7]. The finite-order sequence, as well as the bicomplex, is discussed in [18]. We, however, have nothing more to say on this aspect of the subject.)

In this paper we make considerable use of certain type $(1,1)$ tensor fields which we denote by $S^{i}$. The prototype for these tensors is the canonical almost-tangent structure on a tangent bundle, obtained by composing the tangent projection with the canonical isomorphism of a vector space with its tangent space at any point. Considerable use has been made of this operator over the years in the study of Lagrangian mechanics. There is a similar canonical structure on any higher-order tangent bundle [2], and there are also families of such structures on bundles of (higher-order) velocities [3]. With some additional data, it is possible to construct similar structures on jet bundles, and [11] describes how to associate a tensor $S^{\omega}$ with any closed 1 -form $\omega$ on the base of any fibred manifold.

In much the greater part of the paper we consider fibred manifolds of the specific type $\pi: E \rightarrow \mathbb{R}^{m}$, so that in effect we fix the independent variables. We can therefore work with the tensor $S^{d x^{i}}$ on $J^{\infty} \pi$ where $x^{i}$ are the canonical coordinates on $\mathbb{R}^{m}$; this is the tensor denoted by $S^{i}$. In this particular context, our constructions are all defined 
globally. For a more general fibred manifold $\pi: E \rightarrow M$ and a chart $\left(U, x^{i}\right)$ on $M$, such tensors may be defined locally on $\pi_{\infty}^{-1}(U)$ and were used implicitly in [16] to construct a (local) homotopy operator for the variational bicomplex (see [12, Section 7.3] for an explicit description using $S^{i}$ ). Note that both the homotopy operators we construct in the present paper are different from this earlier one, although one of the resulting projection maps (giving a representation of the Euler-Lagrange complex) turns out to be the well-known 'interior Euler operator'. We consider the effect of taking a general manifold $M$ as the space of independent variables rather than $\mathbb{R}^{m}$ with its canonical coordinate system in the final section of the paper.

The structure of this paper is as follows. In Section 2 we describe the variational bicomplex and recall the basic properties of some geometric operators on $J^{\infty} \pi$. In Section 3 we use these constructions to define the two homotopy operators for the bicomplex, and in Section 4 we construct the representations of the Euler-Lagrange complex. The two versions of the Helmholtz-Sonin conditions are obtained in Section 5. The final section is devoted to a discussion of our results.

\section{Preliminaries}

Let $\pi: E \rightarrow \mathbb{R}^{m}$ be a fibred manifold. We consider the infinite jet manifold $J^{\infty} \pi$; this is an infinite-dimensional Fréchet manifold [12] but the difficulties sometimes associated with such manifolds will not concern us: all our calculations could be repeated, at the cost of dealing with more complicated formulæ, by using objects defined on finite-order jet bundles or along the projection maps between them.

Our notation mostly follows that of [13], which (together with [12]) we suggest as useful references for this section. We denote the canonical coordinates on $\mathbb{R}^{m}$ by $x^{i}$, and of course these are globally defined. Local coordinates on the fibres of $\pi$ will be denoted by $u^{\alpha}$, and jet coordinates by $u_{I}^{\alpha}$. Here, $I, J, \ldots$ are 'symmetric' multi-indices, and are elements of $\mathbb{N}^{m}$. The $i$ th component of $I$ will be denoted by $I(i)$, and the multi-index with $I(i)=1, I(j)=0$ for $j \neq i$ by $1_{i}$. We write $|I|=I(1)+I(2)+\cdots+I(m)$ for the length of $I$, and $I !=I(1) ! I(2) ! \cdots I(m) !$ for its factorial.

We use the summation convention for summation over repeated indices $i, j, \ldots$ (which index the base coordinates), and $\alpha, \beta, \ldots$ (which index the fibre coordinates); however, we indicate sums involving multi-indices explicitly.

In adopting the multi-index convention described above we follow $[3,5,13,18]$ for example. The alternative, adopted by Anderson in [1] for instance, is to use a symbol such as $I$ to stand simply for a collection of ordinary indices: $I=i_{1} i_{2} \ldots i_{k}$. The ratio $|I| ! / I !$, often called the weight of $I$, appears frequently in carrying out translations between the two conventions: it is used when converting a sum over a multi-index into a sum over the corresponding individual indices, so that if some object $\psi(I)$ depends on the multi-index 
$I$ then

$$
\sum_{|I|=r} \frac{|I| !}{I !} \psi(I)=\sum_{i_{1}, i_{2}, \ldots, i_{r}} \psi\left(1_{i_{1}}+1_{i_{2}}+\ldots+1_{i_{r}}\right) .
$$

The following result is an immediate consequence of this observation.

Lemma 1. For any quantitiy $\psi(I)$ depending on the multi-index $I$, and any positive integer $r$,

$$
\sum_{|J|=r+1} \frac{|J| !}{J !} \psi(J)=\sum_{i} \sum_{|I|=r} \frac{|I| !}{I !} \psi\left(I+1_{i}\right)
$$

We use this lemma several times below.

For any variables $\xi^{1}, \xi^{2}, \ldots, \xi^{m}$, we write $\xi^{I}$ for the monomial $\left(\xi^{1}\right)^{I(1)}\left(\xi^{2}\right)^{I(2)} \cdots\left(\xi^{m}\right)^{I(m)}$. The following version of the binomial theorem holds: if $\zeta^{i}=\xi^{i}+\eta^{i}$,

$$
\zeta^{I}=\sum_{J+K=I} \frac{I !}{J ! K !} \xi^{J} \eta^{K}
$$

The power notation extends to any $m$ objects which have a commutative multiplication rule defined on them, such as pairwise commuting operators. Thus with $\partial_{i}=\partial / \partial x^{i}$ we have

$$
\partial_{I}=\frac{\partial^{|I|}}{\left(\partial x^{1}\right)^{I(1)}\left(\partial x^{2}\right)^{I(2)} \cdots\left(\partial x^{m}\right)^{I(m)}},
$$

which gives a simple example of this usage.

We set

$$
\frac{d}{d x^{i}}=\frac{\partial}{\partial x^{i}}+\sum_{I} u_{I+1_{i}}^{\alpha} \frac{\partial}{\partial u_{I}^{\alpha}}
$$

(the $i$ th total derivative), and denote by $d_{i}$ the corresponding operator in the FrölicherNijenhuis calculus, that is, the corresponding Lie derivative. Of course, $d_{i}$ and $d_{j}$ commute, so we may define $d_{I}$ as explained above. We set

$$
\theta_{I}^{\alpha}=d u_{I}^{\alpha}-u_{I+1_{i}}^{\alpha} d x^{i}
$$

(a contact 1-form). We have $d_{i}\left(d x^{j}\right)=0$ and $d_{i} \theta_{I}^{\alpha}=\theta_{I+1_{i}}^{\alpha}$.

The construction of the type $(1,1)$ tensors $S^{i}$ was described in the Introduction. We shall in fact need only the coordinate representation of such tensors, which is given by

$$
S^{i}=\sum_{I}(I(i)+1) \theta_{I}^{\alpha} \otimes \frac{\partial}{\partial u_{I+1_{i}}^{\alpha}} .
$$

Note that $S^{i}\left(d x^{j}\right)=0$. Since $S^{i}$ and $S^{j}$ commute we can define $S^{I}$ (as a type $(1,1)$ tensor), and we find that

$$
S^{I}=\sum_{J} \frac{(I+J) !}{J !} \theta_{J}^{\alpha} \otimes \frac{\partial}{\partial u_{I+J}^{\alpha}} .
$$


We take

$$
S^{0}=\sum_{J} \theta_{J}^{\alpha} \otimes \frac{\partial}{\partial u_{J}^{\alpha}} ;
$$

this type $(1,1)$ tensor, commonly known as the jet bundle contact structure, differs from the identity by $d x^{i} \otimes d / d x^{i}$; acting on vector fields, it is projection onto the vertical along the total derivatives, and so will be written $\mathcal{P}_{V}$. It is easy to show from the formula above that

$$
\mathcal{L}_{d / d x^{i}} S^{I}=-I(i) S^{I-1_{i}},
$$

with the proviso that if $I(i)=0$ then the right-hand side is zero (even though $I-1_{i}$ is undefined).

In the Frölicher-Nijenhuis calculus, with each type $(1,1)$ tensor $S^{j}$ there is associated a derivation of degree 0 and type $i$, denoted by $i_{S^{j}}$. Moreover, since $S^{j}$ and $S^{k}$ commute (as type $(1,1)$ tensors) the corresponding derivations commute. It follows that with any multi-index $I$ we can associate two operators on forms: on the one hand, the composite operator obtained by applying the rule for formation of powers, namely

$$
\left(i_{S^{1}}\right)^{I(1)}\left(i_{S^{2}}\right)^{I(2)} \cdots\left(i_{S^{m}}\right)^{I(m)} ;
$$

on the other, the derivation of type $i$ associated with the type $(1,1)$ tensor $S^{I}$. In general, these are distinct; indeed, the first is not usually a derivation. One could say that, so far as action on forms is concerned, there is an ambiguity in the meaning of $S^{I}$. Now our homotopy operators make use in their definition of the operation of $S^{I}$ on forms. It is a surprising and interesting fact that either interpretation gives a homotopy operator, if one chooses certain constant coefficients appropriately.

The strict Frölicher-Nijenhuis notation can become rather cumbersome. We therefore distinguish the two possibilities notationally as follows: we write $\widetilde{S}^{I}$ to stand for the product of derivations of type $i$, while we re-use the symbol $S^{I}$ to represent the derivation of type $i$ determined by the type $(1,1)$ tensor field $S^{I}$. Notice that as operators on forms, $\widetilde{S}^{0}=$ id, while $S^{0}$ is the derivation of type $i$ corresponding to $\mathcal{P}_{V}$. Of course $\widetilde{S}^{i}=S^{i}$ for a single index $i$, and $\widetilde{S}^{I}=S^{I}$ when acting on a 1 -form.

We now come to the definition of the variational bicomplex. As before, we consider a fibration $\pi: E \rightarrow \mathbb{R}^{m}$ and let $\Omega^{r, s}$ denote the module of $(r+s)$-forms on $J^{\infty} \pi$ containing $r$ contact components and $s$ horizontal components (semi-basic over $M$ ); that is to say, $\Omega^{r, s}$ is spanned by the forms

$$
\theta_{I_{1}}^{\alpha_{1}} \wedge \theta_{I_{2}}^{\alpha_{2}} \wedge \cdots \wedge \theta_{I_{r}}^{\alpha_{r}} \wedge d x^{i_{1}} \wedge d x^{i_{2}} \wedge \cdots \wedge d x^{i_{s}} .
$$

Now $d\left(\Omega^{r, s}\right) \subset \Omega^{r, s+1} \oplus \Omega^{r+1, s}$. We can therefore define maps $d_{H}: \Omega^{r, s} \rightarrow \Omega^{r, s+1}$ and $d_{V}: \Omega^{r, s} \rightarrow \Omega^{r+1, s}$, the horizontal and vertical differentials, such that $d=d_{H}+d_{V}$. These maps satisfy $d_{H}^{2}=d_{V}^{2}=d_{H} \circ d_{V}+d_{V} \circ d_{H}=0$. They are determined by their actions on functions, basic coordinate 1 -forms and contact 1 -forms. For $d_{H}$ we have

$$
d_{H} f=\left(d_{i} f\right) d x^{i}, \quad d_{H}\left(d x^{i}\right)=0, \quad d_{H}\left(\theta_{I}^{\alpha}\right)=-\theta_{I+1_{i}}^{\alpha} \wedge d x^{i} .
$$


It follows that for any $\omega \in \Omega^{r, s}$ we can write

$$
d_{H} \omega=(-1)^{r+s}\left(d_{i} \omega\right) \wedge d x^{i} .
$$

For $d_{V}$, on the other hand,

$$
d_{V} f=\sum_{I} \frac{\partial f}{\partial u_{I}^{\alpha}} \theta_{I}^{\alpha}, \quad d_{V}\left(d x^{i}\right)=d_{V}\left(\theta_{I}^{\alpha}\right)=0 .
$$

The horizontal differential is an invariant object incorporating the total derivative operators on jet bundles. In the context of finite-order jet bundles it would map forms on $J^{k} \pi$ to forms on $J^{k+1} \pi$, but when considering $J^{\infty} \pi$ it is a mapping between forms on the same manifold. On the other hand, the vertical differential has many of the properties of the ordinary exterior derivative on the fibres of $\pi$. Using these operators we construct the variational bicomplex, whose diagram appears as Figure 1 below (where $\Omega^{r}\left(\mathbb{R}^{m}\right.$ ) denotes the module of $r$-forms on $\mathbb{R}^{m}$ ). Note that in this diagram the squares involving both $d_{H}$ and $d_{V}$ are not commutative but anti-commutative. The formula $d_{H} \circ d_{V}+d_{V} \circ d_{H}=0$ arises naturally in the definition of these operators, and it seems appropriate that it should to continue to hold in the diagram even if a little violence is done to the concept of a commutative diagram as a result. It can easily be corrected for by the introduction of some judicious minus signs, as is done for example in [18].

We have written $\Xi^{r}$ for the quotient $\Omega^{r, m} / d_{H}\left(\Omega^{r, m-1}\right)$, and $p_{r}$ for the projection. The operator $\delta_{V}: \Xi^{r} \rightarrow \Xi^{r+1}$ is induced from $d_{V}$ by the projections: $\delta_{V} \circ p_{r}=p_{r+1} \circ d_{V}$; it satisfies $\delta_{V}^{2}=0$. In fact all the rows and columns of the diagram are locally exact. The local exactness of the rows is a consequence of a version of the Poincare lemma, together with the fact that for $\omega \in \Omega^{0, s}, d_{V} \omega=0$ is the necessary and sufficient condition for $\omega$ to be (the pull-back of) an $s$-form on $\mathbb{R}^{m}$. The local exactness of the columns, for $s<m$, follows from the existence of homotopy operators, two examples of which will be exhibited later in this paper; indeed, since the base is $\mathbb{R}^{m}$ with its canonical coordinates our homotopy operators are globally defined and the columns with $r>0$ are therefore globally exact. The rest follows by standard arguments.

The Euler-Lagrange complex is

$$
0 \longrightarrow \mathbb{R} \longrightarrow \Omega^{0,0} \stackrel{d_{H}}{\longrightarrow} \cdots \stackrel{d_{H}}{\longrightarrow} \Omega^{0, m} \stackrel{p_{1} \circ d_{V}}{\longrightarrow} \Xi^{1} \stackrel{\delta_{V}}{\longrightarrow} \Xi^{2} \stackrel{\delta_{V}}{\longrightarrow} \ldots
$$

It too is everywhere locally exact. The most important part of this, so far as we are concerned, is the portion

$$
\Omega^{0, m-1} \stackrel{d_{H}}{\longrightarrow} \Omega^{0, m} \stackrel{p_{1} \circ d_{V}}{\longrightarrow} \Xi^{1} \stackrel{\delta_{V}}{\longrightarrow} \Xi^{2} .
$$

The map $p_{1} \circ d_{V}: \Omega^{0, m} \rightarrow \Xi^{1}$ is the Euler-Lagrange operator. The image $d_{H}\left(\Omega^{0, m-1}\right) \subset$ $\Omega^{0, m}$ can be identified with the total divergences, that is, the forms

$$
\frac{d f_{i}}{d x^{i}} d x^{1} \wedge d x^{2} \wedge \cdots \wedge d x^{m}
$$




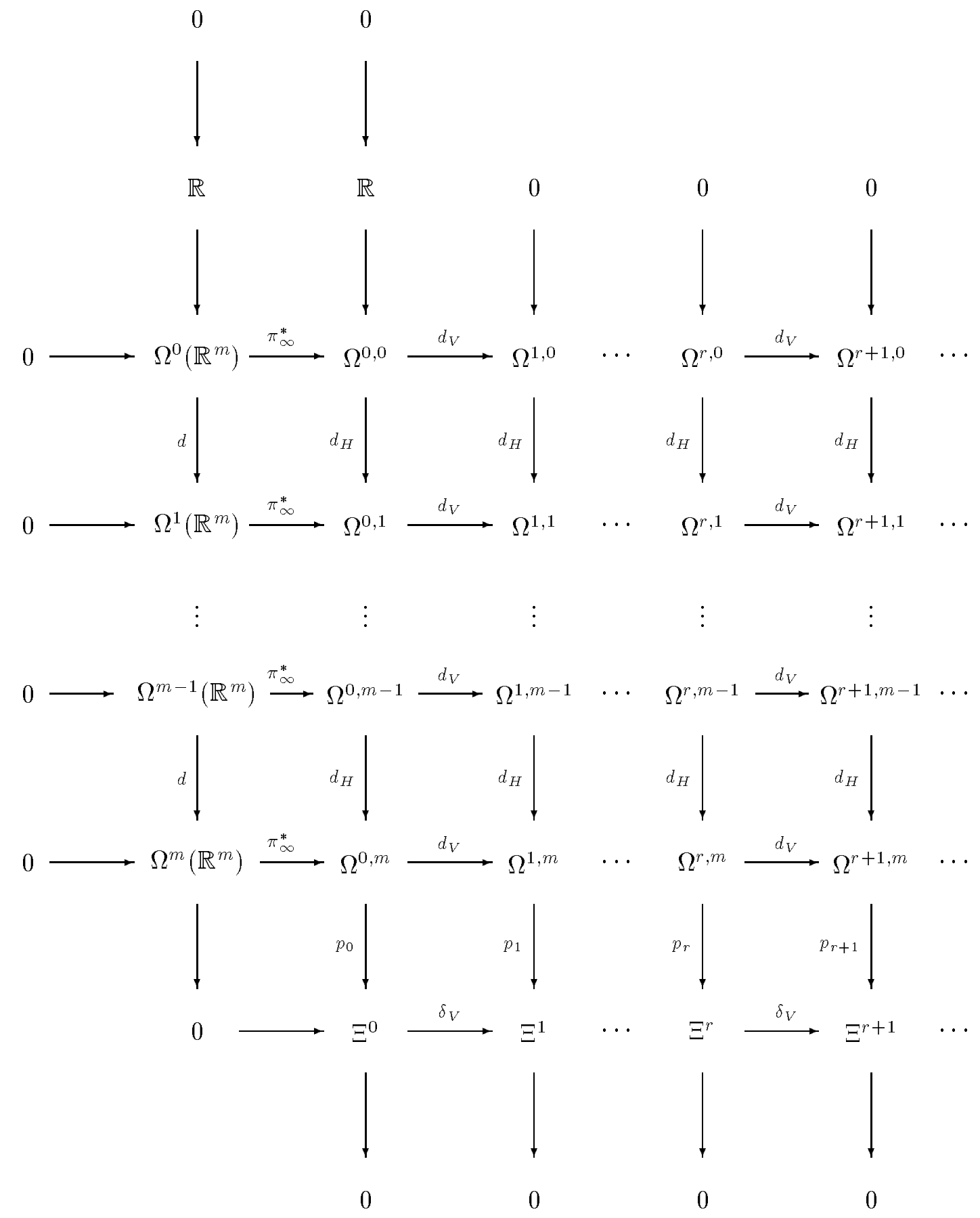

Figure 1: The variational bicomplex 
$\Xi^{1}$ corresponds to the so-called source forms; and $\delta_{V}: \Xi^{1} \rightarrow \Xi^{2}$ is the Helmholtz-Sonin operator, whose kernel consists of those source forms which are variational, that is, which can be identified locally as the Euler-Lagrange form of some Lagrangian.

The Euler-Lagrange complex is in part defined in terms of quotient spaces; for computational purposes it is desirable to have a more explicit representation, and we now address the question of how this may be achieved. The following remarks, though expressed in terms of the variational bicomplex, are in fact quite general and make no appeal to the way that bicomplex is constructed.

Suppose (as we shall eventually show to be the case) that for each $r>0$ we have an $\mathbb{R}$-linear map $\Pi: \Omega^{r, m} \rightarrow \Omega^{r, m}$ such that $\Pi \circ d_{H}: \Omega^{r, m-1} \rightarrow \Omega^{r, m}$ is the zero map, and for every $\omega \in \Omega^{r, m}$ there is some $\phi \in \Omega^{r, m-1}$ such that $\omega=\Pi(\omega)+d_{H}(\phi)$. It is easy to show that

1. each $\Pi$ is a projection operator: $\Pi^{2}=\Pi$;

2. the image of $\Pi$ in $\Omega^{r, m}$, say $\mathcal{E}^{r}$, is complementary to the image of $d_{H}$, and so isomorphic to $\Xi^{r}$; for $\omega \in \Omega^{r, m}$, we have $\omega \in \mathcal{E}^{r}$ if and only if $\Pi(\omega)=\omega$;

3. if we set $\Pi\left(d_{V}(\omega)\right)=\delta(\Pi(\omega))$ then $\delta$ is well-defined as a map $\mathcal{E}^{r} \rightarrow \mathcal{E}^{r+1}$, and $\delta^{2}=0$.

One way of finding such a map $\Pi$ is to use a homotopy operator. Suppose that we know a homotopy operator for the columns with $r>0$, say $P$; that is, $P: \Omega^{r, s} \rightarrow \Omega^{r, s-1}$ for $1 \leq s \leq m$, and $P \circ d_{H}+d_{H} \circ P: \Omega^{r, s} \rightarrow \Omega^{r, s}$ for $1 \leq s<m$ is the identity. (We should, of course, require also that $P \circ d_{H}: \Omega^{r, 0} \rightarrow \Omega^{r, 0}$ be the identity, but it is easy to see how to extend the definitions so that we may use the standard formula for this degenerate situation too.)

Now consider $\Pi=\mathrm{id}-d_{H} \circ P: \Omega^{r, m} \rightarrow \Omega^{r, m}$. Then for any $\phi \in \Omega^{r, m-1}$, we have

$$
\Pi\left(d_{H}(\phi)\right)=d_{H}(\phi)-d_{H}\left(P\left(d_{H}(\phi)\right)=d_{H}(\phi)-d_{H}\left(\phi-d_{H}(P(\phi))=0 ;\right.\right.
$$

and for any $\omega \in \Omega^{r, m}$ we have $\omega=\Pi(\omega)+d_{H}(P(\omega))$. Thus $\Pi$ has the properties discussed above, so that a homotopy operator for the columns gives us a representation of the Euler-Lagrange complex, in the form of spaces $\mathcal{E}^{r} \subset \Omega^{r, m}$ representing $\Xi^{r}$, and maps $\delta: \mathcal{E}^{r} \rightarrow \mathcal{E}^{r+1}$ representing $\delta_{V}$.

As in effect we remarked earlier, one of the main points of interest addressed in this paper is the fact that since we can construct two different homotopy operators for the columns, we have two distinct ways of defining projection operators $\Pi$. The corresponding two representations of the Euler-Lagrange complex are of course isomorphic, in an obvious sense. In fact the two spaces $\mathcal{E}^{1}$ are identical, and so is the map $\Omega^{0, m} \rightarrow \mathcal{E}^{1}$. Since this is the operator that produces the Euler-Lagrange equations from a given Lagrangian, this is all to the good. However, differences set in after this point. As a result, $\delta\left(\mathcal{E}^{1}\right) \subset \Omega^{2, m}$ differs in the two cases, and so the condition for $\varepsilon \in \mathcal{E}^{1}$ to be variational, namely $\delta \varepsilon=0$, 
takes different forms. That is to say, the two representations produce different versions of the Helmholtz-Sonin conditions.

\section{The homotopy operators}

For $\omega \in \Omega^{r, s}$ with $r>0$ we define

$$
\left.\left.P(\omega)=\frac{d}{d x^{j}}\right\lrcorner P_{(r, s)}^{j}(\omega), \quad \widetilde{P}(\omega)=\frac{d}{d x^{j}}\right\lrcorner \widetilde{P}_{(r, s)}^{j}(\omega),
$$

where $P_{(r, s)}^{j}: \Omega^{r, s} \rightarrow \Omega^{r, s}$ is given by

$$
P_{(r, s)}^{j}=\sum_{J} \frac{(-1)^{|J|}(m-s) !|J| !}{r(m-s+|J|+1) ! J !} d_{J} \circ S^{J+1_{j}}
$$

and $\widetilde{P}_{(r, s)}^{j}: \Omega^{r, s} \rightarrow \Omega^{r, s}$ by

$$
\tilde{P}_{(r, s)}^{j}=\sum_{J} \frac{(-1)^{|J|}(m-s) !|J| !}{r^{|J|+1}(m-s+|J|+1) ! J !} d_{J} \circ \widetilde{S}^{J+1_{j}} .
$$

We claim that for $s<m$ both $P$ and $\widetilde{P}$ are homotopy operators for $d_{H}$.

Theorem 1. For $\omega \in \Omega^{r, s}$, where $s<m$ and $r>0$,

$$
\left(d_{H} P+P d_{H}\right)(\omega)=\omega=\left(d_{H} \widetilde{P}+\widetilde{P} d_{H}\right)(\omega) .
$$

Proof. We give the argument for $P$ in detail, and then explain how it may be modified so as to apply to $\widetilde{P}$.

First, two remarks about hook and wedge. For any $\omega \in \Omega^{r, s}$ we have

$$
\left.\left.\left(\frac{d}{d x^{j}}\right\lrcorner \omega\right) \wedge d x^{i}=\frac{d}{d x^{j}}\right\lrcorner\left(\omega \wedge d x^{i}\right)-(-1)^{r+s} \delta_{j}^{i} \omega
$$

since $\lrcorner$ is an (anti-)derivation with respect to $\wedge$. Furthermore,

$$
\left.\frac{d}{d x^{i}}\right\lrcorner\left(\omega \wedge d x^{i}\right)=(-1)^{r+s}(m-s) \omega .
$$

To see this, consider $\omega=\phi \wedge d x^{i_{1}} \wedge d x^{i_{2}} \wedge \cdots \wedge d x^{i_{s}}$ with $i_{1}<i_{2}<\cdots<i_{s}$, where $\phi \in \Omega^{r, 0}$. Then

$$
\begin{aligned}
\left.\frac{d}{d x^{i}}\right\lrcorner\left(\omega \wedge d x^{i}\right) & \left.=(-1)^{r} \phi \wedge\left(\sum_{i \notin\left\{i_{1}, i_{2}, \ldots, i_{s}\right\}} \frac{d}{d x^{i}}\right\lrcorner\left(d x^{i_{1}} \wedge d x^{i_{2}} \wedge \cdots \wedge d x^{i_{s}} \wedge d x^{i}\right)\right) \\
& =(-1)^{r} \phi \wedge\left(\sum_{i \notin\left\{i_{1}, i_{2}, \ldots, i_{s}\right\}}(-1)^{s} d x^{i_{1}} \wedge d x^{i_{2}} \wedge \cdots \wedge d x^{i_{s}}\right) \\
& =(-1)^{r+s}(m-s) \omega .
\end{aligned}
$$


From the formula for $\mathcal{L}_{d / d x^{i}} S^{I}$ it follows that (as a commutator of derivations)

$$
\left[d_{i}, S^{I-}\right]=-I(i) S^{I-1_{i}} .
$$

Here, $S^{0}$ is the derivation of type $i$ defined by $\mathcal{P}_{V}$. In particular, acting on $\Omega^{r, s}$ we have $S^{0}=r$ id.

We now turn to the proof of the homotopy property. We have

$$
\begin{aligned}
& \left(d_{H} P+P d_{H}\right)(\omega) \\
& \left.\left.\quad=(-1)^{r+s-1} d_{i}\left(\frac{d}{d x^{j}}\right\lrcorner P_{(r, s)}^{j}(\omega)\right) \wedge d x^{i}+(-1)^{r+s} \frac{d}{d x^{j}}\right\lrcorner P_{(r, s+1)}^{j}\left(d_{i} \omega \wedge d x^{i}\right) \\
& \left.\left.=(-1)^{r+s-1}\left(\frac{d}{d x^{j}}\right\lrcorner d_{i} P_{(r, s)}^{j}(\omega)\right) \wedge d x^{i}+(-1)^{r+s} \frac{d}{d x^{j}}\right\lrcorner\left(P_{(r, s+1)}^{j}\left(d_{i} \omega\right) \wedge d x^{i}\right) \\
& \left.=d_{i} P_{(r, s)}^{i}(\omega)+(-1)^{r+s} \frac{d}{d x^{j}}\right\lrcorner\left(\left(P_{(r, s+1)}^{j} d_{i}-d_{i} P_{(r, s)}^{j}\right)(\omega) \wedge d x^{i}\right),
\end{aligned}
$$

so the main part of the proof is the calculation of $P_{(r, s+1)}^{j} \circ d_{i}-d_{i} \circ P_{(r, s)}^{j}$. The first step is to commute the $d_{i}$ through the $S^{J+1_{j}}$ terms in $P_{(r, s+1)}^{j} \circ d_{i}$. From the commutator formula we have

$$
S^{J+1_{j}} \circ d_{i}=d_{i} \circ S^{J+1_{j}}+J(i) S^{J+1_{j}-1_{i}}+\delta_{i}^{j} S^{J} .
$$

It follows that

$$
\begin{aligned}
& P_{(r, s+1)}^{j} \circ d_{i} \\
& \quad=\sum_{J} \frac{(-1)^{|J|}(m-s-1) !|J| !}{r(m-s+|J|) ! J !}\left(d_{J+1_{i}} \circ S^{J+1_{j}}+J(i) d_{J} \circ S^{J+1_{j}-1_{i}}+\delta_{i}^{j} d_{J} \circ S^{J}\right) .
\end{aligned}
$$

In the term containing $J(i)$ the summation effectively starts from $J=1_{i}$, and we may change the summation variable from $J$ to $J-1_{i}$ to get

$$
\begin{aligned}
& \sum_{J \geq 1_{i}} \frac{(-1)^{|J|}(m-s-1) !|J| !}{r(m-s+|J|) ! \cdot J !} J(i) d_{J} \circ S^{J+1_{j}-1_{i}} \\
& \quad=-\sum_{J} \frac{(-1)^{|J|}(m-s-1) !(|J|+1) !}{r(m-s+|J|+1) ! J !} d_{J+1_{i}} \circ S^{J+1_{j}}
\end{aligned}
$$


Then

$$
\begin{aligned}
P_{(r, s+1)}^{j} \circ d_{i}-d_{i} \circ P_{(r, s)}^{j}= & \sum_{J} \frac{(-1)^{|J|}(m-s-1) !|J| !}{r(m-s+|J|) ! \cdot J !} d_{J+1_{i}} \circ S^{J+1_{j}} \\
& -\sum_{J} \frac{(-1)^{|J|}(m-s-1) !(|J|+1) !}{r(m-s+|J|+1) ! \cdot J !} d_{J+1_{i}} \circ S^{J+1_{j}} \\
& -\sum_{J} \frac{(-1)^{|J|}(m-s) !|J| !}{r(m-s+|J|+1) ! J !} d_{J+1_{i}} \circ S^{J+1_{j}} \\
& +\delta_{i}^{j} \sum_{J} \frac{(-1)^{|J|}(m-s-1) !|J| !}{r(m-s+|J|) ! J !} d_{J} \circ S^{J} .
\end{aligned}
$$

Inspection of the coefficients reveals that the first three terms cancel, and we are left with

$$
\begin{aligned}
& \left.(-1)^{r+s} \frac{d}{d x^{j}}\right\lrcorner\left(\left(P_{(r, s+1)}^{j} d_{i}-d_{i} P_{(r, s)}^{j}\right)(\omega) \wedge d x^{i}\right) \\
& \left.=(-1)^{r+s} \frac{d}{d x^{i}}\right\lrcorner\left(\sum_{J} \frac{(-1)^{|J|}(m-s-1) !|J| !}{r(m-s+|J|) ! J !} d_{J} S^{J} \omega \wedge d x^{i}\right) \\
& \quad=\sum_{J} \frac{(-1)^{|J|}(m-s) !|J| !}{r(m-s+|J|) ! J !} d_{J} S^{J} \omega .
\end{aligned}
$$

Thus

$$
\begin{gathered}
\left.\left(d_{H} P+P d_{H}\right)(\omega)=d_{i} P_{(r, s)}^{i}(\omega)+(-1)^{r+s} \frac{d}{d x^{j}}\right\lrcorner\left(\left(P_{(r, s+1)}^{j} d_{i}-d_{i} P_{(r, s)}^{j}\right)(\omega) \wedge d x^{i}\right) \\
=\sum_{I} \frac{(-1)^{|I|}(m-s) !|I| !}{r(m-s+|I|+1) ! I !} d_{I+1_{i}} S^{I+1_{i}} \omega+\sum_{I} \frac{(-1)^{|I|}(m-s) !|I| !}{r(m-s+|I|) ! I !} d_{I} S^{I} \omega .
\end{gathered}
$$

There is of course an implied summation over $i$ in the first term in this formula. If we apply Lemma 1 with

$$
\psi(I)=\frac{(-1)^{|I|}(m-s) !}{r(m-s+|I|) !} d_{I} S^{I} \omega
$$

we see that the contributions to the first sum in the formula with $|I|=s$ cancel with the contributions to the second sum with $|I|=s+1$. All that remains is the term in the second sum with $I=0$, which is just

$$
\frac{1}{r} S^{0} \omega=\omega .
$$

Thus $\left(d_{H} P+P d_{H}\right)(\omega)=\omega$, as asserted.

The calculation for $\widetilde{P}$ is very similar. The main difference is in the commutator, which in this case is calculated as follows. On $\Omega^{r, s}$ we have $\left[d_{i}, S^{j}\right]=-r \delta_{i}^{j} \mathcal{P}_{V}$. Now for any 
operators $A, B$ and $C$ we have $[A, B C]=[A, B] C+B[A, C]$, so that in computing $\left[d_{i}, \widetilde{S}^{I}\right]$ we need only consider the terms in $\widetilde{S}^{I}$ involving $S^{i}$; that is to say

$$
\left[d_{i}, \widetilde{S}^{I-}\right]=\left(S^{1}\right)^{I(1)}\left(S^{2}\right)^{I(2)} \cdots\left[d_{i},\left(S^{i}\right)^{I(i)}\right] \cdots\left(S^{n}\right)^{I(n)} .
$$

Moreover, $\left[d_{i},\left(S^{i}\right)^{I(i)}\right]=-r I(i)\left(S^{i}\right)^{I(i)-1}$, and so $\left[d_{i}, \widetilde{S}^{I}\right]=-r I(i) \widetilde{S}^{I-1_{i}}$. It follows that

$$
\left[d_{i}, \widetilde{S}^{J+1_{j}}\right]=-r\left(J(i) \widetilde{S}^{J+1_{j}-1_{i}}+\delta_{i}^{j} \widetilde{S}^{J}\right)
$$

The occurence of the extra coefficient $r$ is accommodated by the difference in the coefficient in the definition of $\widetilde{P}$ compared to $P$. See [14] for the detailed calculation in a different but related context.

\section{Representations of the Euler-Lagrange complex}

In the case of the homotopy operator $P$ the projection operator on $\Omega^{r, m}$ is $\Pi=\mathrm{id}-d_{H} \circ P$. Let us denote an element of $\Omega^{r, m}$ by $\phi \wedge d^{m} x, \phi \in \Omega^{r, 0}$, and set

$$
\Pi\left(\phi \wedge d^{m} x\right)=\Pi_{r}(\phi) \wedge d^{m} x .
$$

We derive two explicit formulæ for $\Pi_{r}$.

Theorem 2. For $\phi \in \Omega^{r, 0}$

$$
\left.\Pi_{r}(\phi)=\frac{1}{r} \sum_{I}(-1)^{|I|} \frac{1}{I !} d_{I} S^{I}(\phi)=\frac{1}{r} \theta^{\alpha} \wedge \sum_{I}(-1)^{|I|} d_{I}\left(\frac{\partial}{\partial u_{I}^{\alpha}}\right\lrcorner \phi\right) .
$$

Proof. We have

$$
\begin{aligned}
d_{H}\left(P\left(\phi \wedge d^{m} x\right)\right) & \left.=(-1)^{r+m-1} d_{i}\left(\frac{d}{d x^{j}}\right\lrcorner\left(P_{(r, m)}^{j}(\phi) \wedge d^{m} x\right)\right) \wedge d x^{i} \\
& =d_{j} P_{(r, m)}^{j}(\phi) \wedge d^{m} x .
\end{aligned}
$$

But

$$
P_{(r, m)}^{j}=\sum_{J} \frac{(-1)^{|J|}|J| !}{r(|J|+1) ! J !} d_{J} \circ S^{J+1_{j}}
$$

and so

$$
\Pi_{r}=\mathrm{id}-\sum_{J} \frac{(-1)^{|J|}|J| !}{r(|J|+1) ! J !} d_{J+1_{j}} \circ S^{J+1_{j}} .
$$

By Lemma 1 again,

$$
-\sum_{J} \frac{(-1)^{|J|}|J| !}{r(|J|+1) ! \cdot J !} d_{J+1_{j}} \circ S^{J+1_{\jmath}}=\sum_{|J|>0} \frac{(-1)^{|J|}|J| !}{r(|J|) ! J !} d_{J} \circ S^{J}
$$


and so

$$
\Pi_{r}=\frac{1}{r} \sum_{J}(-1)^{|J|} \frac{1}{J !} d_{J} \circ S^{J} .
$$

The alternative formula for $\Pi_{r}$ is obtained as follows. In the Frölicher-Nijenhuis calculus the derivation of type $i$ corresponding to the type $(1,1)$ tensor $\alpha \otimes V$ (where $\alpha$ is a 1 -form and $V$ a vector field) is just $\left.i_{\alpha \otimes V} \phi=\alpha \wedge(V\lrcorner \phi\right)$. Using the expression for $S^{I}$ as a sum of tensor products, and remembering that $S^{I}$ stands for $i_{S^{I}}$, we find that

$$
\left.\Pi_{r}(\phi)=\frac{1}{r} \sum_{I, J}(-1)^{|I|} \frac{(I+J) !}{I ! J !} d_{I}\left(\theta_{J}^{\alpha} \wedge\left(\frac{\partial}{\partial u_{I+J}^{\alpha}}\right\lrcorner \phi\right)\right)
$$

We now use the Leibniz rule, and the fact that $d_{K} \theta_{J}^{\alpha}=\theta_{J+K}^{\alpha}$, to obtain

$$
\begin{aligned}
\Pi_{r}(\phi) & \left.=\frac{1}{r} \sum_{I, J}(-1)^{|I|} \frac{(I+J) !}{I ! J !} \sum_{K+L=I} \frac{(K+L) !}{K ! L !} \theta_{J+K}^{\alpha} \wedge d_{L}\left(\frac{\partial}{\partial u_{J+K+L}^{\alpha}}\right\lrcorner \phi\right) \\
& =\frac{1}{r} \sum_{J, K, L}(-1)^{|K+L|} \frac{(J+K+L) !}{J ! K ! L !} \theta_{J+K}^{\alpha} \wedge d_{L}\left(\frac{\partial}{\left.\left.\partial u_{J+K+L}^{\alpha}\right\lrcorner \phi\right)}\right. \\
& \left.=\frac{1}{r} \sum_{L, M}(-1)^{|L|} \frac{(L+M) !}{L ! M !}\left(\sum_{J+K=M}(-1)^{|K|} \frac{M !}{J ! K !}\right) \theta_{M}^{\alpha} \wedge d_{L}\left(\frac{\partial}{\partial u_{L+M}^{\alpha}}\right\lrcorner \phi\right) .
\end{aligned}
$$

By the multi-index binomial theorem, for $M \neq 0$

$$
\sum_{J+K=M}(-1)^{|K|} \frac{M !}{J ! K !}=(1-1)^{|M|}=0 .
$$

Thus only the terms with $M=0$ contribute to the sum; it follows that

$$
\left.\Pi_{r}(\phi)=\frac{1}{r} \theta^{\alpha} \wedge \sum_{I}(-1)^{|I|} d_{I}\left(\frac{\partial}{\partial u_{I}^{\alpha}}\right\lrcorner \phi\right) .
$$

This latter expression identifies $\Pi_{r}$ with the interior Euler operator (see [18]), and so the $\mathcal{E}^{r}$ are the spaces of variational forms (or functional forms as they are called in [1]).

In the case of $\widetilde{\Pi}$ we have only one formula, corresponding to the first of those for $\Pi$.

\section{Theorem 3.}

$$
\widetilde{\Pi}_{r}=\sum_{I}\left(-\frac{1}{r}\right)^{|I|} \frac{1}{I !} d_{I} \circ \widetilde{S}^{I}
$$

The derivation of this formula is similar to that of the one for $\Pi_{r}$.

Next, we give a simple characterisation of the image $\widetilde{\mathcal{E}}^{r}$ of the projection operator $\widetilde{\Pi}$. 
Theorem 4. The subspace $\widetilde{\mathcal{E}}^{r}$ of $\Omega^{r, m}$ consists of forms $\phi \wedge d^{m} x$ such that $S^{i}(\phi)=0$, $i=1,2, \ldots, m$.

Proof. Clearly if $\phi$ satisfies the given condition then $\widetilde{S}^{I}(\phi)=0$ for all $I \neq 0$, and so $\widetilde{\Pi}_{r}(\phi)=\phi$. For the converse we use the fact that $S^{i} \circ d_{j}=d_{j} \circ S^{i}+r \delta_{j}^{i}$ (when acting on an element of $\left.\Omega^{r, s}\right)$, which generalises to $S^{i} \circ d_{I}=d_{I} \circ S^{i}+r I(i) d_{I-1_{i}}$. Then

$$
\begin{aligned}
S^{i} \circ \tilde{\Pi}_{r} & =\sum_{I}\left(-\frac{1}{r}\right)^{|I|} \frac{1}{I !} S^{i} \circ d_{I} \circ \widetilde{S}^{I} \\
& =\sum_{I}\left(-\frac{1}{r}\right)^{|I|} \frac{1}{I !}\left(d_{I} \circ \widetilde{S}^{I+1_{i}}+r I(i) d_{I-1_{i}} \circ \widetilde{S}^{I}\right) \\
& =\sum_{I}\left(-\frac{1}{r}\right)^{|I|} \frac{1}{I !} d_{I} \circ \widetilde{S}^{I+1_{i}}-\sum_{I \geq 1_{i}}\left(-\frac{1}{r}\right)^{|I|-1} \frac{1}{\left(I-1_{i}\right) !} d_{I-1_{i}} \circ \widetilde{S}^{I} \\
& =0 .
\end{aligned}
$$

Thus if $\widetilde{\Pi}_{r}(\phi)=\phi$, then $S^{i}(\phi)=0$.

When $r=1, S^{I}=\widetilde{S}^{I}$, and so $\Pi_{1}=\widetilde{\Pi}_{1}$, and $\mathcal{E}^{1}=\widetilde{\mathcal{E}}^{1}$. The latter consists of all forms $\phi \wedge d^{m} x$ with $\phi \in \Omega^{1,0}$ and $S^{i}(\phi)=0$; it follows that $\mathcal{E}^{1}$ is spanned by the forms $\theta^{\alpha} \wedge d^{m} x$. Moreover, the Euler-Lagrange map $\Omega^{0, m} \rightarrow \mathcal{E}^{1}$ is given by $L d^{m} x \mapsto \Pi_{1}\left(d_{V} L\right) \wedge d^{m} x$, and

$$
\Pi_{1}\left(d_{V} L\right)=\Pi_{1}\left(\sum_{I} \frac{\partial L}{\partial u_{I}^{\alpha}} \theta_{I}^{\alpha}\right)=\sum_{I}(-1)^{|I|} d_{I}\left(\frac{\partial L}{\partial u_{I}^{\alpha}}\right) \theta^{\alpha} .
$$

It is a consequence of Theorem 4 that the vector spaces $\widetilde{\mathcal{E}}^{r}$ are modules over the ring of functions on $J^{\infty} \pi$. In contrast, the spaces $\mathcal{E}^{r}$ are not modules when $r \geq 2$ : to see an example of this, take the fibred manifold $\pi: \mathbb{R} \times \mathbb{R}^{2} \rightarrow \mathbb{R}$ and consider the 3 -form $\phi \wedge d x$ where

$$
\phi=\left(d u_{(2)}^{1} \wedge d u^{2}+d u^{1} \wedge d u_{(2)}^{2}\right) \in \Omega^{2,0} .
$$

A simple calculation shows that $d_{H}(P(\phi \wedge d x))=0$, so that $\phi \wedge d x \in \mathcal{E}^{2}$. On the other hand, taking $\psi=u^{1} \phi$ we find that

$$
d_{H}(P(\psi \wedge d x))=-d_{H}\left(u_{(1)}^{1} d u^{1} \wedge d u^{2}\right) \neq 0
$$

so that $\psi \wedge d x \notin \mathcal{E}^{2}$. Indeed, characterizing $\mathcal{E}^{r}$ for $r \geq 2$ is known to be a difficult problem; we discuss the case $r=2$ later.

\section{The Helmholtz-Sonin conditions}

We now compute $\delta \varepsilon$ and $\tilde{\delta} \varepsilon$ for an arbitrary source form $\varepsilon=\varepsilon_{\alpha} \theta^{\alpha} \wedge d^{m} x$ (where $\delta=\Pi \circ d_{V}$ and $\tilde{\delta}=\widetilde{\Pi} \circ d_{V}$ ). We start with $\delta \varepsilon$. 


\section{Theorem 5.}

$$
\delta \varepsilon=-\frac{1}{2} \sum_{I} \mathcal{H}_{\alpha \beta}^{I} \theta^{\alpha} \wedge \theta_{I}^{\beta} \wedge d^{m} x
$$

where

$$
\mathcal{H}_{\alpha \beta}^{I}=\frac{\partial \varepsilon_{\alpha}}{\partial u_{I}^{\beta}}-\sum_{J}(-1)^{|I+J|} \frac{(I+J) !}{I ! J !} d_{J}\left(\frac{\partial \varepsilon_{\beta}}{\partial u_{I+J}^{\alpha}}\right)
$$

Proof. We have

$$
\delta \varepsilon=\Pi\left(d_{V} \varepsilon\right)=\frac{1}{2} \sum_{I}(-1)^{|I|} \frac{1}{I !} d_{I} S^{I}\left(\sum_{J} \frac{\partial \varepsilon_{\alpha}}{\partial u_{J}^{\beta}} \theta_{J}^{\beta} \wedge \theta^{\alpha} \wedge d^{m} x\right) .
$$

Now

$$
S^{I}\left(\theta_{J}^{\beta}\right)=\left\{\begin{array}{cl}
\frac{J !}{(J-I) !} \theta_{J-I}^{\beta} & \text { if } J \geq I \\
0 & \text { otherwise. }
\end{array}\right.
$$

In particular, $S^{I}\left(\theta^{\beta}\right)=0$, unless $I=0: S^{0}\left(\theta^{\beta}\right)=\theta^{\beta}$. Thus

$$
\begin{aligned}
\delta \varepsilon & =\sum_{J} \frac{\partial \varepsilon_{\alpha}}{\partial u_{J}^{\beta}} \theta_{J}^{\beta} \wedge \theta^{\alpha} \wedge d^{m} x+\frac{1}{2} \sum_{|I|>0} \sum_{J}(-1)^{|I|} \frac{(I+J) !}{I ! J !} d_{I}\left(\frac{\partial \varepsilon_{\alpha}}{\partial u_{I+J}^{\beta}} \theta_{J}^{\beta} \wedge \theta^{\alpha} \wedge d^{m} x\right) \\
& =\frac{1}{2} \sum_{J} \frac{\partial \varepsilon_{\alpha}}{\partial u_{J}^{\beta}} \theta_{J}^{\beta} \wedge \theta^{\alpha} \wedge d^{m} x+\frac{1}{2} \sum_{I, J}(-1)^{|I|} \frac{(I+J) !}{I ! J !} d_{I}\left(\frac{\partial \varepsilon_{\alpha}}{\partial u_{I+J}^{\beta}} \theta_{J}^{\beta} \wedge \theta^{\alpha} \wedge d^{m} x\right) .
\end{aligned}
$$

We evaluate the second term on the right. Of course $d_{K}\left(d^{m} x\right)=0$ for $K>0$, and

$$
\begin{aligned}
& \sum_{I, J}(-1)^{|I|} \frac{(I+J) !}{I ! J !} d_{I}\left(\frac{\partial \varepsilon_{\alpha}}{\partial u_{I+J}^{\beta}} \theta_{J}^{\beta} \wedge \theta^{\alpha}\right) \\
& \quad=\sum_{I, J}(-1)^{|I|} \frac{(I+J) !}{I ! J !} \sum_{K+L+M=I} \frac{(K+L+M) !}{K ! L ! M !} d_{K}\left(\frac{\partial \varepsilon_{\alpha}}{\partial u_{I+J}^{\beta}}\right) d_{L}\left(\theta_{J}^{\beta}\right) \wedge d_{M}\left(\theta^{\alpha}\right) \\
& =\sum_{J, K, L, M}(-1)^{|K+L+M|} \frac{(J+K+L+M) !}{J ! K ! L ! M !} d_{K}\left(\frac{\partial \varepsilon_{\alpha}}{\partial u_{J+K+L+M}^{\beta}}\right) \theta_{J+L}^{\beta} \wedge \theta_{M}^{\alpha} \\
& =\sum_{K, M, N}(-1)^{|K+M|} \frac{(K+M+N) !}{K ! M ! N !}\left(\sum_{J+L=N}(-1)^{|L|} \frac{N !}{J ! L !}\right) d_{K}\left(\frac{\partial \varepsilon_{\alpha}}{\partial u_{K+M+N}^{\beta}}\right) \theta_{N}^{\beta} \wedge \theta_{M}^{\alpha} .
\end{aligned}
$$

Using the multi-index binomial theorem again we obtain

$$
\delta \varepsilon=\frac{1}{2} \sum_{J} \frac{\partial \varepsilon_{\alpha}}{\partial u_{J}^{\beta}} \theta_{J}^{\beta} \wedge \theta^{\alpha} \wedge d^{m} x-\frac{1}{2} \sum_{I, J}(-1)^{|I+J|} \frac{(I+J) !}{I ! J !} d_{I}\left(\frac{\partial \varepsilon_{\beta}}{\partial u_{I+J}^{\alpha}}\right) \theta_{J}^{\beta} \wedge \theta^{\alpha} \wedge d^{m} x .
$$


Our result agrees verbatim with [5] (mutatis mutandis). For purposes of comparison with the formulæ in other articles, such as $[6,9]$, it is useful to write

$$
\mathcal{H}_{\alpha \beta}^{I}=\frac{\partial \varepsilon_{\alpha}}{\partial u_{I}^{\beta}}-(-1)^{|I|} \frac{\partial \varepsilon_{\beta}}{\partial u_{I}^{\alpha}}-\sum_{|J|>0}(-1)^{|I+J|} \frac{(I+J) !}{I ! J !} d_{J}\left(\frac{\partial \varepsilon_{\beta}}{\partial u_{I+J}^{\alpha}}\right) .
$$

Note in particular that only the terms not involving $d_{J}$ are collectively skew or symmetric in $\alpha$ and $\beta$; the rest involve just $\varepsilon_{\beta}$. The Helmholtz-Sonin conditions, in this version, are

$$
\mathcal{H}_{\alpha \beta}^{I}=0, \quad I \neq 0 ; \quad \mathcal{H}_{\alpha \beta}-\mathcal{H}_{\beta \alpha}=0 .
$$

(We believe that insufficient attention has sometimes been paid to the seeming anomaly that arises for $I=0$.)

The Helmholtz-Sonin conditions derived from $\tilde{\delta}$ are interestingly quite different in appearance. In deriving them we take advantage of the fact that $\widetilde{\mathcal{E}}^{2}$ consists of $(m+2)$-forms annihilated by $S^{i}$ for all $i$. We begin by using this fact to obtain an explicit basis of $\widetilde{\mathcal{E}}^{2}$.

Lemma 2. For any $I, \alpha, \beta$ set

$$
\Theta_{I}^{\alpha \beta}=\sum_{J+K=I}(-1)^{|K|} \frac{I !}{J ! K !} \theta_{J}^{\alpha} \wedge \theta_{K}^{\beta} \wedge d^{m} x
$$

(Notice that $\Theta_{I}^{\alpha \beta}$ is 'graded skew-symmetric' in $\alpha$ and $\beta$ : that is, $\Theta_{I}^{\beta \alpha}=-(-1)^{|I|} \Theta_{I}^{\alpha \beta}$.) The set of forms $\left\{\Theta_{I}^{\alpha \beta}: \alpha<\beta\right.$ for $|I|$ even, $\alpha \leq \beta$ for $|I|$ odd $\}$ is a basis for $\mathcal{E}^{2}$.

Proof. We show first that $\Theta_{I}^{\alpha \beta} \in \mathcal{E}^{2}$. Remembering that $S^{i}$ is a derivation of degree 0 , we have

$$
S^{i}\left(\Theta_{I}^{\alpha \beta}\right)=\sum_{J+K=I}(-1)^{|K|} \frac{I !}{J ! K !}\left(J(i) \theta_{J-1_{i}}^{\alpha} \wedge \theta_{K}^{\beta} \wedge d^{m} x+K(i) \theta_{J}^{\alpha} \wedge \theta_{K-1_{i}}^{\beta} \wedge d^{m} x\right) .
$$

If $I(i)=0$ then $S^{i}\left(\Theta_{I}^{\alpha \beta}\right)=0$. So suppose that $I(i) \neq 0$, and consider the coefficient of the term in $S^{i}\left(\Theta_{I}^{\alpha \beta}\right)$ involving $\theta_{L}^{\alpha} \wedge \theta_{M}^{\beta} \wedge d^{m} x$, where $L$ and $M$ are fixed multi-indices with $L+M=I-1_{i}$. Such a term has two antecedents: $\theta_{L+1_{i}}^{\alpha} \wedge \theta_{M}^{\beta} \wedge d^{m} x$ and $\theta_{L}^{\alpha} \wedge \theta_{M+1_{i}}^{\beta} \wedge d^{m} x$; their joint contribution to the coefficient is

$$
(-1)^{|M|} \frac{I !}{\left(L+1_{i}\right) ! M !}(L(i)+1)+(-1)^{|M|+1} \frac{I !}{L !\left(M+1_{i}\right) !}(M(i)+1)=0 .
$$

Thus $S^{i}\left(\Theta_{I}^{\alpha \beta}\right)=0$ for all $i$, as asserted. It is not difficult to show, by an extension of the same technique, that the $\Theta_{I}^{\alpha \beta}$ span $\widetilde{\mathcal{E}}^{2}$. Finally, it is clear that the $\Theta_{I}^{\alpha \beta}$ for different $I$ are linearly independent (making allowance for the fact that they are graded skew in $\alpha$ and $\beta$ ).

We next compute $\tilde{\delta} \varepsilon$ for an arbitrary source form $\varepsilon=\varepsilon_{\alpha} \theta^{\alpha} \wedge d^{m} x$. 


\section{Theorem 6.}

$$
\tilde{\delta} \varepsilon=-\frac{1}{2} \sum_{I} \tilde{\mathcal{H}}_{\alpha \beta}^{I} \Theta_{I}^{\alpha \beta}
$$

where

$$
\tilde{\mathcal{H}}_{\alpha \beta}^{I}=\sum_{J}\left(-\frac{1}{2}\right)^{|I+J|} \frac{(I+J) !}{I ! J !} d_{J}\left(\frac{\partial \varepsilon_{\alpha}}{\partial u_{I+J}^{\beta}}-(-1)^{|I|} \frac{\partial \varepsilon_{\beta}}{\partial u_{I+J}^{\alpha}}\right)
$$

Note especially that like $\Theta_{I}^{\alpha \beta}$, but in sharp contrast to $\mathcal{H}_{\alpha \beta}^{I}, \widetilde{\mathcal{H}}_{\alpha \beta}^{I}$ is graded skewsymmetric in $\alpha$ and $\beta$.

Proof. We have

$$
\tilde{\delta} \varepsilon=\widetilde{\Pi}\left(d_{V} \varepsilon\right)=\sum_{I}\left(-\frac{1}{2}\right)^{|I|} \frac{1}{I !} d_{I} \widetilde{S}^{I}\left(\sum_{J} \frac{\partial \varepsilon_{\alpha}}{\partial u_{J}^{\beta}} \theta_{J}^{\beta} \wedge \theta^{\alpha} \wedge d^{m} x\right) .
$$

Since $S^{i}\left(\theta^{\alpha}\right)=0$, the evaluation of $\widetilde{S}^{I}\left(\theta_{J}^{\beta} \wedge \theta^{\alpha} \wedge d^{m} x\right)$ is particularly simple:

$$
\widetilde{S}^{I}\left(\theta_{J}^{\beta} \wedge \theta^{\alpha} \wedge d^{m} x\right)=\widetilde{S}^{I}\left(\theta_{J}^{\beta}\right) \wedge \theta^{\alpha} \wedge d^{m} x=S^{I}\left(\theta_{J}^{\beta}\right) \wedge \theta^{\alpha} \wedge d^{m} x
$$

The initial stages of the calculation are consequently similar to those in Theorem 5 , and we find the following expression for $\tilde{\delta} \varepsilon$ :

$$
\sum_{K, M, N}\left(-\frac{1}{2}\right)^{|K+M|} \frac{(K+M+N) !}{K ! M ! N !} \sum_{J+L=N}\left(\left(-\frac{1}{2}\right)^{|L|} \frac{N !}{J ! L !}\right) d_{K}\left(\frac{\partial \varepsilon_{\alpha}}{\partial u_{K+M+N}^{\beta}}\right) \theta_{N}^{\beta} \wedge \theta_{M}^{\alpha} \wedge d^{m} x .
$$

This time the multi-index binomial theorem gives

$$
\sum_{J+L=N}\left(-\frac{1}{2}\right)^{|L|} \frac{N !}{J ! L !}=\left(1-\frac{1}{2}\right)^{|N|}=\left(\frac{1}{2}\right)^{|N|},
$$

and the subsequent steps are quite different. In fact

$$
\tilde{\delta} \varepsilon=-\sum_{K, M, N}\left(-\frac{1}{2}\right)^{|K+M+N|}(-1)^{|N|} \frac{(K+M+N) !}{K ! M ! N !} d_{K}\left(\frac{\partial \varepsilon_{\alpha}}{\partial u_{K+M+N}^{\beta}}\right) \theta_{M}^{\alpha} \wedge \theta_{N}^{\beta} \wedge d^{m} x
$$

but equally

$$
\tilde{\delta} \varepsilon=-\sum_{K, M, N}\left(-\frac{1}{2}\right)^{|K+M+N|}(-1)^{|M|} \frac{(K+M+N) !}{K ! M ! N !} d_{K}\left(\frac{\partial \varepsilon_{\beta}}{\partial u_{K+M+N}^{\alpha}}\right) \theta_{N}^{\beta} \wedge \theta_{M}^{\alpha} \wedge d^{m} x
$$


(just changing the names of some of the summation indices). So we can write

$$
\begin{aligned}
\tilde{\delta} \varepsilon=-\frac{1}{2} \sum_{K, M, N}\left(-\frac{1}{2}\right)^{|K+M+N|} \frac{(K+M+N) !}{K ! M ! N !} \times \\
\quad \times d_{K}\left((-1)^{|N|} \frac{\partial \varepsilon_{\alpha}}{\partial u_{K+M+N}^{\beta}}-(-1)^{|M|} \frac{\partial \varepsilon_{\beta}}{\partial u_{K+M+N}^{\alpha}}\right) \theta_{M}^{\alpha} \wedge \theta_{N}^{\beta} \wedge d^{m} x \\
=-\frac{1}{2} \sum_{K, M, N}\left(-\frac{1}{2}\right)^{|K+M+N|}(-1)^{|N|} \frac{(K+M+N) !}{K ! M ! N !} \times \\
\quad \times d_{K}\left(\frac{\partial \varepsilon_{\alpha}}{\partial u_{K+M+N}^{\beta}}-(-1)^{|M+N|} \frac{\partial \varepsilon_{\beta}}{\partial u_{K+M+N}^{\alpha}}\right) \theta_{M}^{\alpha} \wedge \theta_{N}^{\beta} \wedge d^{m} x \\
=-\frac{1}{2} \sum_{K, P}\left(-\frac{1}{2}\right)^{|K+P|} \frac{(K+P) !}{K ! P !} d_{K}\left(\frac{\partial \varepsilon_{\alpha}}{\partial u_{K+P}^{\beta}}-(-1)^{|P|} \frac{\partial \varepsilon_{\beta}}{\partial u_{K+P}^{\alpha}}\right) \times \\
\quad \times \sum_{M+N=P}(-1)^{|N|} \frac{P !}{M ! N !} \theta_{M}^{\alpha} \wedge \theta_{N}^{\beta} \wedge d^{m} x,
\end{aligned}
$$

as required.

The Helmholtz-Sonin conditions, in this version, are just $\widetilde{\mathcal{H}}_{\alpha \beta}^{I}=0$.

The two versions of the Helmholtz-Sonin conditions, though superficially so different, must of course at some deeper level be equivalent. We next investigate the relationship between them. For this purpose it will be convenient to refer to the first version (involving $\mathcal{H}_{\alpha \beta}^{I}$ ) as the standard version, since indeed it is the version most usually found in the literature; we call the second version the new version. In fact the relationship in question is a bit subtle, because, as Anderson says in [1], there are 'certain interdependencies amongst the [standard] Helmholtz conditions'. The precise meaning of this somewhat obscure utterance will become clear in the following discussion.

Before dealing with the conditions themselves, we shall examine the relation between the spaces $\mathcal{E}^{2}$ and $\widetilde{\mathcal{E}}^{2}$. These two spaces must clearly be isomorphic, and in fact $\left.\Pi\right|_{\widetilde{\mathcal{E}}^{2}}: \widetilde{\mathcal{E}}^{2} \rightarrow$ $\mathcal{E}^{2}$ and $\left.\widetilde{\Pi}\right|_{\mathcal{E}^{2}}: \mathcal{E}^{2} \rightarrow \widetilde{\mathcal{E}}^{2}$ are mutually inverse isomorphisms. We know a great deal about $\widetilde{\mathcal{E}}^{2}$; we shall use our information, together with $\Pi$, to investigate $\mathcal{E}^{2}$.

We shall use Roman letters to indicate general elements of the spaces $\mathcal{E}^{2}$ and $\widetilde{\mathcal{E}}^{2}$, retaining calligraphic letters for the particular case where the elements arise as the images of Helmholtz-Sonin maps. Any $\widetilde{H} \in \widetilde{\mathcal{E}}^{2}$ can be written $\widetilde{H}=\sum_{I} \widetilde{H}_{\alpha \beta}^{I} \Theta_{I}^{\alpha \beta}$, where the implied summation on $\alpha$ and $\beta$ is taken over all $\alpha, \beta$ and the coefficients $\widetilde{H}_{\alpha \beta}^{I}$ are graded skew-symmetric, i.e. $\widetilde{H}_{\beta \alpha}^{I}=-(-1)^{|I|} \widetilde{H}_{\alpha \beta}^{I}$. The calculation of $\Pi(\widetilde{H})$ follows a by now familiar pattern, and we won't repeat it; the result is

$$
\Pi(\widetilde{H})=\sum_{I} H_{\alpha \beta}^{I} \theta^{\alpha} \wedge \theta_{I}^{\beta} \wedge d^{m} x, \quad \text { where } H_{\alpha \beta}^{I}=(-2)^{|I|} \sum_{J}(-1)^{|J|} \frac{(I+J) !}{I ! J !} d_{J} \widetilde{H}_{\alpha \beta}^{I+J} .
$$


But the $\widetilde{H}_{\alpha \beta}^{I}$ are graded skew-symmetric, and therefore

$$
H_{\beta \alpha}^{I}=-2^{|I|} \sum_{J} \frac{(I+J) !}{I ! J !} d_{J} \widetilde{H}_{\alpha \beta}^{I+J} .
$$

We shall show below that, as a result, the $H_{\alpha \beta}^{I}$ must satisfy

$$
H_{\beta \alpha}^{I}=-(-1)^{|I|} \sum_{J}(-1)^{|J|} \frac{(I+J) !}{I ! J !} d_{J} H_{\alpha \beta}^{I+J} .
$$

This is our equivalent of a condition given by Anderson for a 2 -form to be a functional form (see [1] Eq.3.14, for 2-forms). We call it Anderson's condition.

Consider on the other hand an $(m+2)$-form $H=\sum_{I} H_{\alpha \beta}^{I} \theta^{\alpha} \wedge \theta_{I}^{\beta} \wedge d^{m} x$ where the coefficients satisfy Anderson's condition. Define

$$
\widetilde{H}_{\alpha \beta}^{I}=\left(-\frac{1}{2}\right)^{|I|} \sum_{J}\left(-\frac{1}{2}\right)^{|J|} \frac{(I+J) !}{I ! J !} d_{J} H_{\alpha \beta}^{I+J}
$$

again, we show below that the $\widetilde{H}_{\alpha \beta}^{I}$ are graded skew-symmetric, and if we set $\widetilde{H}=$ $\sum_{I} \widetilde{H}_{\alpha \beta}^{I} \Theta_{I}^{\alpha \beta} \in \widetilde{\mathcal{E}}^{2}$ we have $H=\Pi(\widetilde{H})$.

To prove the assertions just made, we shall let $\mathbf{U}$ be the space of families of functions on $J^{\infty} \pi$ indexed by the multi-index $I$, that is, the space of maps $\mathbb{N}^{m} \rightarrow C^{\infty}\left(J^{\infty} \pi\right)$; for $U \in \mathbf{U}$ the value of $U$ at $I$ is denoted by $U^{I}$ (rather than $U(I)$ ). For $p \in \mathbb{R}$, define $\mathcal{D}(p): \mathbf{U} \rightarrow \mathbf{U}$ by

$$
(\mathcal{D}(p) U)^{I}=\sum_{J} p^{|J|} \frac{(I+J) !}{I ! J !} d_{J} U^{I+J}=U^{I}+\sum_{|J|>0} p^{|J|} \frac{(I+J) !}{I ! J !} d_{J} U^{I+J}
$$

so that, in particular, $\mathcal{D}(0)$ is the identity. Then $\mathcal{D}(p) \mathcal{D}(q)=\mathcal{D}(p+q)$ :

$$
\begin{aligned}
(\mathcal{D}(p) \mathcal{D}(q) U)^{I} & =\sum_{J} p^{|J|} \frac{(I+J) !}{I ! J !} d_{J}\left(\sum_{K} q^{|K|} \frac{(I+J+K) !}{(I+J) ! K !} d_{K} U^{I+J+K}\right) \\
& =\sum_{J, K} p^{|J|} q^{|K|} \frac{(I+J+K) !}{I ! J ! K !} d_{J+K} U^{I+J+K} \\
& =\sum_{L} \frac{(I+L) !}{I ! L !}\left(\sum_{J+K=L} p^{|J|} q^{|K|} \frac{(J+K) !}{J ! K !}\right) d_{L} U^{I+L} \\
& =\sum_{L}(p+q)^{|L|} \frac{(I+L) !}{I ! L !} d_{L} U^{I+L},
\end{aligned}
$$

by the binomial theorem. Notice that $\mathcal{D}(-p)=(\mathcal{D}(p))^{-1}$. 
We now return to the formulæ

$$
H_{\alpha \beta}^{I}=(-2)^{|I|} \sum_{J}(-1)^{|J|} \frac{(I+J) !}{I ! J !} d_{J} \widetilde{H}_{\alpha \beta}^{I+J}
$$

and

$$
\widetilde{H}_{\alpha \beta}^{I}=\left(-\frac{1}{2}\right)^{|I|} \sum_{J}\left(-\frac{1}{2}\right)^{|J|} \frac{(I+J) !}{I ! J !} d_{J} H_{\alpha \beta}^{I+J},
$$

relating coefficients of elements of $\mathcal{E}^{2}$ and $\widetilde{\mathcal{E}}^{2}$. If we set $\widehat{H}_{\alpha \beta}^{I}=\left(-\frac{1}{2}\right)^{|I|} H_{\alpha \beta}^{I}$ these formulæ can be written

$$
\widehat{H}_{\alpha \beta}=\mathcal{D}(-1)\left(\widetilde{H}_{\alpha \beta}\right), \quad \widetilde{H}_{\alpha \beta}=\mathcal{D}(1)\left(\hat{H}_{\alpha \beta}\right),
$$

with $\widetilde{H}_{\alpha \beta}, \widehat{H}_{\alpha \beta} \in \mathbf{U}$, making it obvious that they are inverse. Moreover, in deriving the consequences for $H_{\alpha \beta}^{I}$ of the graded skew-symmetry of $\widetilde{H}_{\alpha \beta}^{I}$ we found in effect that $\bar{H}_{\alpha \beta}^{I}=\frac{1}{2}^{|I|} H_{\alpha \beta}^{I}$ satisfies $\bar{H}_{\beta \alpha}=-\mathcal{D}(1)\left(\widetilde{H}_{\alpha \beta}\right)$. It follows that $\bar{H}_{\beta \alpha}=-\mathcal{D}(2)\left(\hat{H}_{\alpha \beta}\right)$, which when written out is the Anderson condition

$$
H_{\beta \alpha}^{I}=-(-1)^{|I|} \sum_{J}(-1)^{|J|} \frac{(I+J) !}{I ! J !} d_{J} H_{\alpha \beta}^{I+J} .
$$

It follows that the set of forms $H=\sum_{I} H_{\alpha \beta}^{I} \theta^{\alpha} \wedge \theta_{I}^{\beta} \wedge d^{m} x$ where the coefficients satisfy Anderson's condition is the image of $\widetilde{\mathcal{E}}^{2}$ under $\Pi$; but this is just $\mathcal{E}^{2}$.

Now for any source form $\varepsilon, \delta \varepsilon=-\frac{1}{2} \sum_{I} \mathcal{H}_{\alpha \beta}^{I} \theta^{\alpha} \wedge \theta_{I}^{\beta} \wedge d^{m} x$. But of course $\delta \varepsilon \in \mathcal{E}^{2}$, so the coefficients must satisfy Anderson's condition. In this sense the standard HelmholtzSonin conditions are not independent; the role of Anderson's condition in the definition of $\mathcal{E}^{2}$ is the source of the interdependencies between them.

In the course of the discussion we have established explicit relationships between the two forms of Helmholtz-Sonin expressions. In particular,

$$
\mathcal{H}_{\alpha \beta}^{I}=\left(-\frac{1}{2}\right)^{|I|} \sum_{J}\left(-\frac{1}{2}\right)^{|J|} \frac{(I+J) !}{I ! J !} d_{J} \widetilde{\mathcal{H}}_{\alpha \beta}^{I+J},
$$

a formula which can be established also by a straightforward calculation using the explicit expressions for $\mathcal{H}_{\alpha \beta}^{I}$ and $\widetilde{\mathcal{H}}_{\alpha \beta}^{I}$ in terms of $\varepsilon$ given earlier.

Since there are no a priori interdependencies among the new Helmholtz-Sonin conditions, they are easier to work with than the standard ones. Consider for example the case when there is a single dependent variable. The new Helmholtz-Sonin conditions vanish identically when $|I|$ is even and give

$$
\sum_{J}\left(-\frac{1}{2}\right)^{|J|} \frac{(I+J) !}{I ! J !} d_{J}\left(\frac{\partial \varepsilon}{\partial u_{I+J}}\right)=0
$$


when $|I|$ is odd. This can be written

$$
\frac{\partial \varepsilon}{\partial u_{I}}+\sum_{|J|>0}\left(-\frac{1}{2}\right)^{|J|} \frac{(I+J) !}{I ! J !} d_{J}\left(\frac{\partial \varepsilon}{\partial u_{I+J}}\right)=0 .
$$

Anderson obtains an expression of a similar form, but with less straightforward coefficients, for this case (Eq.3.19 of [1]) by a complicated series of eliminations from the standard conditions.

\section{Discussion}

In this paper we have constructed two different versions of the Helmholtz-Sonin conditions, and demonstrated the relationship between them. One version, involving $\mathcal{H}_{\alpha \beta}^{I}$, is well-known in the literature; the other, involving $\widetilde{\mathcal{H}}_{\alpha \beta}^{I}$, is new. In some respects, the new conditions have better properties than the older ones. We have, however, chosen a particular type of fibred manifold, where the base is $\mathbb{R}^{m}$ with its canonical coordinate system. In such a context our constructions are global, and the expressions $\mathcal{H}_{\alpha \beta}^{I}, \widetilde{\mathcal{H}}_{\alpha \beta}^{I}$ transform correctly under changes of fibre coordinates as the components of differential forms. There are applications where such a context is appropriate, for instance in mechanics with a 1-dimensional base $\mathbb{R}$ and a given time coordinate $t$. But in other applications we would wish to take a general base manifold $M$, and it is well-known that a transformation of the independent variables in a variational problem can destroy covariance (see, for instance, the remarks in [10]). We can be sure only that our operators $P$ and $\widetilde{P}$ will be local homotopy operators in this more general case (though it is known that for $r>0$ and $s<m$ the columns are globally exact: see [18]).

It is, indeed, the case that covariance fails for $\widetilde{\mathcal{H}}_{\alpha \beta}^{I}$, as we now show. Taking $m=1$, $n=2$ and the 3 -form $\phi \wedge d x$ with

$$
\phi=d u_{x x} \wedge d v \wedge d x \in \Omega^{2,0}
$$

(we must modify the notation here to allow for the fact that we are going to change the independent variables) a simple calculation gives

$$
\widetilde{\Pi}^{x}(\phi \wedge d x)=\frac{1}{4}\left(d u_{x x} \wedge d v-2 d u_{x} \wedge d v_{x}+d u \wedge d v_{x x}\right) \wedge d x
$$

where the superscript indicates that the calculation of $\widetilde{\Pi}$ has been performed using $x$ as the independent variable. On the other hand, taking a new coordinate $y$ on $M$ with $x=e^{y}$, we can write $\widetilde{\Pi}^{x}(\phi \wedge d x)$ in the new coordinates as

$$
\widetilde{\Pi}^{x}(\phi \wedge d x)=\frac{1}{4} e^{-y}\left\{\left(d u_{y y}-d u_{y}\right) \wedge d v-2 d u_{y} \wedge d v_{y}+d u \wedge\left(d v_{y y}-d v_{y}\right)\right\} \wedge d y,
$$

whereas a direct computation gives

$$
\widetilde{\Pi}^{y}(\phi \wedge d y)=\frac{1}{4} e^{-2 y}\left\{d u_{y y} \wedge d v+d u \wedge d v_{y y}-2 d u_{y} \wedge d v_{y}-d u \wedge d v\right\} \wedge d y
$$


showing that the expression for $\widetilde{\Pi}(\phi \wedge d x)$ does not transform correctly under a change of independent variables.

Using the same example, we see also that

$$
\Pi^{x}(\phi \wedge d x)=\frac{1}{2} e^{-y}\left\{\left(d u_{y y}-d u_{y}\right) \wedge d v+d u \wedge\left(d v_{y y}-d v_{y}\right)\right\} \wedge d y=\Pi^{y}(\phi \wedge d y),
$$

and indeed several authors have shown (see [18] and references therein) that the interior Euler operator is globally well-defined under an arbitrary fibred change of coordinates on $\pi: E \rightarrow M$.

In conclusion: the interior Euler operator and the variational (functional) forms which it produces are covariant but awkward to work with, and lead to Helmholtz-Sonin conditions which are inelegant and not independent; our version avoids these difficulties, but at the cost of being dependent on a particular choice of base coordinates.

\section{Acknowledgements}

The first author is a Guest Professor at Ghent University: he is grateful to the Department of Mathematical Physics and Astronomy for its hospitality.

The second author acknowledges the support of grant no. 201/06/0922 for Global Analysis and its Applications from the Czech Science Foundation.

\section{References}

[1] I. M. Anderson: The Variational Bicomplex, http://www .math.usu.edu/“fg_mp/

[2] M. Crampin, W. Sarlet and F. Cantrijn: Higher-order differential equations and higher-order Lagrangian mechanics Math. Proc. Camb. Phil. Soc. 99 (1986) 565587

[3] M. Crampin and D.J. Saunders: The Hilbert-Carathéodory and Poincaré-Cartan forms for higher-order multiple-integral variational problems Houston J. Math. 30 (2004) 657-689

[4] A. Frölicher and A. Nijenhuis: Theory of vector-valued differential forms Nederl. Akad. Wetensch. Proc. A59 (1956) 338-359

[5] I. Kolář and R. Vitolo: On the Helmholtz operator for Euler morphisms Math. Proc. Cam. Phil. Soc. 135 (2003) 277-290

[6] M. Krbek, J. Musilová and J. Kašparová: The variational sequence: local and global properties In: Proceedings of the Seminar on Differential Geometry, ed. Krupka (Mathematical Publications Volume 2, Silesian University in Opava, 2000) 15-38 
[7] M. Krbek and J. Musilová: Representation of the variational sequence by differential forms Acta Appl. Math. 88 (2005) 177-199

[8] D. Krupka: Variational sequences on finite order jet spaces In: Differential Geometry and its Applications (Proceedings of the International Conference, Brno, Czechoslovakia, 1989), ed. Janyška and Krupka (World Scientific, 1990) 236-254

[9] D. Krupka: The Vainberg-Tonti Lagrangian and the Euler-Lagrange mapping In: Differential Geometric Methods in Mechanics and Field Theory, ed. Cantrijn, Crampin and Langerock (Academia Press, Gent, 2007) 81-90

[10] P. J. Olver: Equivalence and the Cartan form Acta Appl. Math. 31 (1993) 99-136

[11] D.J. Saunders: An alternative approach to the Cartan form in Lagrangian field theories J. Phys. A: Math. Gen. 20 (1987) 339-349

[12] D. J. Saunders: The Geometry of Jet Bundles (Cambridge University Press, 1989)

[13] D. J. Saunders: Jet manifolds and natural bundles In: Handbook of Global Analysis, ed. Krupka and Saunders (Elsevier, 2008) 1035-1067

[14] D.J. Saunders: Homogeneous variational complexes and bicomplexes ArXiV:math . $\mathrm{DG} / 0512383$

[15] F. Takens: A global version of the inverse problem to the calculus of variations $J$. Diff. Geom. 14 (1979) 543-562

[16] W.M. Tulczyjew: The Euler-Lagrange resolution In: Lecture Notes in Mathematics 836 (Springer, 1980) 22-48

[17] A. M. Vinogradov: The $\mathcal{C}$-spectral sequence, Lagrangian formalism and conservation laws J. Math. Anal. Appl. 100 (1984) 1-129

[18] R. Vitolo: Variational sequences In: Handbook of Global Analysis, ed. Krupka and Saunders (Elsevier, 2008) 1115-1163 